\title{
On a Griffiths-Harris Conjecture
}

\author{
Ananyo Dan *
}

April 19, 2022

\section{Introduction}

In 1882, M. Noether claimed the following statement which was later proven by Lefschetz: For $d \geq 4$, a very general smooth degree $d$ surface $X$ in $\mathbb{P}^{3}$ has Picard number $\rho(X)=1$. This motivates the definition of the Noether-Lefschetz locus, denoted by $\mathrm{NL}_{d}$ parametrizing the space of smooth degree $d$ surfaces $X$ in $\mathbb{P}^{3}$ with $\rho(X)>1$. One of the interesting problems is to understand the geometry of the Noether-Lefschetz locus. By the Lefschetz $(1,1)$-theorem, we can look at an irreducible component of the Noether-Lefschetz locus locally as a Hodge locus (see [Voi03, §5] for more details). In particular, denote by $U_{d} \subseteq \mathbb{P}\left(H^{0}\left(\mathbb{P}^{3}, \mathcal{O}_{\mathbb{P}^{3}}(\mathrm{~d})\right)\right)$ the open subscheme parametrizing smooth projective hypersurfaces in $\mathbb{P}^{3}$ of degree $d$. Let $\mathcal{X} \stackrel{\pi}{\rightarrow} U_{d}$ be the corresponding universal family. For a given $F \in U_{d}$, denote by $X_{F}$ the surface $X_{F}:=$ $\pi^{-1}(F)$. Let $X \in U_{d}$ and $U \subseteq U_{d}$ be a simply connected neighbourhood of $X$ in $U_{d}$ (under the analytic topology). Then, $\left.\pi\right|_{\pi^{-1}(U)}$ induces a variation of Hodge structure $(\mathcal{H}, \nabla)$ on $U$, where $\mathcal{H}:=R^{2} \pi_{*} \mathbb{Z} \otimes \mathcal{O}_{U}$ and $\nabla$ is the Gauss-Manin connection. Note that $\mathcal{H}$ defines a local system on $U$ whose fiber over a point $F \in U$ is $H^{2}\left(X_{F}, \mathbb{Z}\right)$. Consider a non-zero element $\gamma_{0} \in H^{2}\left(X_{F}, \mathbb{Z}\right) \bigcap H^{1,1}\left(X_{F}, \mathbb{C}\right)$ such that $\gamma_{0} \neq c_{1}\left(\mathcal{O}_{X_{F}}(k)\right)$ for $k \in \mathbb{Z}_{>0}$. This defines a section $\gamma \in(\mathcal{H} \otimes \mathbb{C})(U)$. Let $\bar{\gamma}$ be the image of $\gamma$ in $\mathcal{H} / F^{2}(\mathcal{H} \otimes \mathbb{C})$. The Hodge loci corresponding to $\gamma$,

${ }^{*}$ The author has been supported by the DFG under Grant KL-2244/2 - 1

Humboldt Universität zu Berlin, Institut für Mathematik, Unter den Linden 6, Berlin 10099. e-mail:dan@mathematik.hu-berlin.de

Mathematics Subject Classification: 14C30, 14D07 
denoted $\operatorname{NL}(\gamma)$ is then defined as

$$
\mathrm{NL}(\gamma):=\left\{G \in U \mid \bar{\gamma}_{G}=0\right\}
$$

where $\bar{\gamma}_{G}$ denotes the value at $G$ of the section $\bar{\gamma}$. For an irreducible component $L \subset \mathrm{NL}_{d}$ and $X \in L$, general, we can find $\gamma \in H^{1,1}(X, \mathbb{Z}):=H^{2}(X, \mathbb{Z}) \bigcap H^{1,1}(X, \mathbb{C})$ such that $\overline{\mathrm{NL}(\gamma)}=L$ (the closure taken in $U_{d}$ under Zariski topology).

One of the first results in this direction is due to Green, Griffiths, Voisin and others (Gre89, GH83, Voi88]) which states that for an irreducible component $L$ of the Noether-Lefschetz locus, that for $d \geq 4$,

$$
d-3 \leq \operatorname{codim}\left(L, U_{d}\right) \leq\left(\begin{array}{c}
d-1 \\
3
\end{array}\right)
$$

The upper bound follows easily from the fact that $\operatorname{dim} H^{2,0}(X)=\left(\begin{array}{c}d-1 \\ 3\end{array}\right)$ for any $X \in U_{d}$ (see Voi03, §6]). We say that $L$ is a general component if $\operatorname{codim} L=\left(\begin{array}{c}d-1 \\ 3\end{array}\right)$ and special otherwise. It was proven by Ciliberto, Harris and Miranda CHM88 that for $d \geq 4$, the Noether-Lefschetz locus has infinitely many general components and the union of these components is Zariski dense in $U_{d}$. The guiding principle of much work in the area has been the expectation that special components should be due to the presence of low degree curves. Voisin Voi89] and Green Gre89. independently proved that for $d \geq 5, \operatorname{codim} L=d-3$ if and only if $L$ parametrizes surfaces of degree $d$ containing a line. If $d-3<\operatorname{codim} L \leq 2 d-7$ then $\operatorname{codim} L=2 d-7$ and $L$ parametrizes the surfaces containing a conic. Otwinowska Otw03 proved that for an integer $b>0$ and $d \gg b$ if $\operatorname{codim} L \leq b d$ then $L$ parametrizes surfaces containing a curve of degree at most $b$.

For $r \geq 3$, we define the level $r$-Noether-Lefschetz locus, denoted $\mathrm{NL}_{r, d}$ to be the space parametrizing surfaces with Picard number greater than or equal to $r$. It has been conjectured by Griffiths and Harris in GH83 that for $r<d$, an irreducible component of $\mathrm{NL}_{r, d}$ is of codimension greater than or equal to $(r-1)(d-3)-\left(\begin{array}{c}r-3 \\ 2\end{array}\right)$. Furthermore, the component of $\mathrm{NL}_{r, d}$ parametrizing surfaces containing $r-1$ coplanar lines is of this codimension. In this article we prove (in Theorem 5.11) that:

Theorem 1.1. Let $r \geq 3$ and $d \gg r$. Let $L$ be an irreducible component of $\mathrm{NL}_{r, d}$. Then, $\operatorname{codim} L \geq(r-1)(d-3)-\left(\begin{array}{c}r-3 \\ 2\end{array}\right)$. Furthermore, there exists a component $L$ of $\mathrm{NL}_{r, d}$ of this 
codimension parametrizing surfaces containing $r-1$ coplanar lines.

The techniques used to prove this result is a combination of deformation theory and Hodge theory. Instead of looking at the Hodge locus corresponding to a Hodge class, we study the Hodge locus corresponding to a $\mathbb{Z}$-module of Hodge classes. We then use a result due to Otwinowska, Otw04, Theorem 1], to show that if the codimension of an irreducible component $L$ of $\mathrm{NL}_{r, d}$ is less than or equal to $(r-1)(d-3)-\left(\begin{array}{c}r-3 \\ 2\end{array}\right)$, then for a general $X \in L$ there exists a lattice $\Lambda \subset H^{1,1}(X, \mathbb{Z})$ generated by classes of curves of degree less than or equal to $r-1$ such that $L$ is locally of the form $\mathrm{NL}(\Lambda)$ (see Proposition [5.6), where $\mathrm{NL}(\Lambda)$ is the intersection of $\mathrm{NL}(\gamma)$ for all $\gamma \in \Lambda, \bigcap_{\gamma \in \Lambda} \mathrm{NL}(\gamma)$.

We now use the theory of semi-regularity as introduced in Blo72] to reduce the problem to a question in flag Hilbert schemes. First to fix some notations, for a Hilbert polynomial $P$ for some curve $C$ in $\mathbb{P}^{3}$, we denote by $H_{P}$ the corresponding Hilbert scheme, parametrizing curves (schemes with pure dimension 1) with Hilbert polynomial $P$. Throughout this article we denote by $Q_{d}$ the Hilbert polynomial of a degree $d$ surface in $\mathbb{P}^{3}$. We denote by $H_{P, Q_{d}}$ the corresponding flag Hilbert scheme parametrizing pairs $(C, X)$ such that $C \in H_{P}, X \in H_{Q_{d}}$ and $C \subset X$. A curve $C$ on a smooth surface in $\mathbb{P}^{3}$ is said to be semi-regular if $H^{1}\left(\mathcal{O}_{X}(C)\right)=0$. We prove that

Theorem 1.2. Let $X$ be a smooth surface in $\mathbb{P}^{3}$ of degree $d$ and $C \subset X$, a semi-regular curve with Hilbert polynomial, say $P$. For any irreducible component, $L^{\prime}$ of $\overline{\mathrm{NL}([C])}$ (the closure is taken in $U_{d}$ under Zariski topology) there exists an irreducible component $H^{\prime}$ of $H_{P, Q_{d}}$ containing the pair $(C, X)$ such that $\operatorname{pr}_{2}\left(H^{\prime}\right)_{\text {red }}$ coincides with $L_{\text {red }}^{\prime}$, where $\mathrm{pr}_{2}$ is the second projection map from $H_{P, Q_{d}}$ to $H_{Q_{d}}$. In particular, if $C$ is reduced, connected and $d \geq \operatorname{deg}(C)+4$ then this holds true and the irreducible component $H^{\prime}$ is uniquely determined by $L^{\prime}$.

See Theorem 3.10 and Lemma 3.4 for the precise statements and its proof. Using this proof we further show that under the above bound on the codimension of $L$, the lattice will infact be generated by classes of lines (see Lemma 5.9 ). Finally, we do a computation in Proposition 5.10 , to determine the "arrangement" of these lines which would help us determine the component with the correct codimension.

Acknowledgement: I would like to thank Prof. R. Kloosterman for reading the preliminary version of this article and several helpful discussions. 


\section{Introduction to Noether-Lefschetz locus}

2.1. In this section we recall the basic definitions of Noether-Lefschetz locus. See [Voi02, $§ 9,10]$ and Voi03, $\S 5,6]$ for a detailed presentation of the subject.

Notation 2.2. By a component of $\mathrm{NL}_{d}$, we mean an irreducible component. By a surface we always mean a smooth surface in $\mathbb{P}^{3}$. Denote by $Q_{d}$ the Hilbert polynomial of degree $d$ surfaces in $\mathbb{P}^{3}$. Given, a Hilbert polynomial $P$, denote by $H_{P}$ the corresponding Hilbert scheme and by $H_{P, Q_{d}}$ the corresponding flag Hilbert scheme. Also, for a point $u \in U_{d}$, denote by $X_{u}$ the fiber $\pi^{-1}(u)$.

Notation 2.3. Let $X \in U_{d}$ and $\mathcal{O}_{X}(1)$, the very ample line bundle on $X$ determined by the closed immersion $X \hookrightarrow \mathbb{P}^{3}$ arising (as in [Har77, II.Ex.2.14(b)]) from the graded homomorphism of graded rings $S \rightarrow S /\left(F_{X}\right)$, where $S=\Gamma_{*}\left(\mathcal{O}_{\mathbb{P}^{3}}\right)$ and $F_{X}$ is the defining equations of $X$. Denote by $H_{X}$ the very ample line bundle $\mathcal{O}_{X}(1)$. Note that a very ample line bundle on $X_{u}$ for any $u \in U$ remains very ample in the family $\mathcal{X}$, hence the corresponding cohomology class remains of type $(1,1)$ in $\mathcal{X}$.

2.4. Let $X$ be a surface. The Lefschetz hyperplane section theorem implies,

$$
H^{2}(X, \mathbb{C}) \cong H^{2}(X, \mathbb{C})_{\text {prim }} \oplus \mathbb{C} H_{X}
$$

where $H_{X}$ is the very ample line bundle on $X$ and $H^{2}(X, \mathbb{C})_{\text {prim }}$ is the primitive cohomology. This gives us a natural projection map from $H^{2}(X, \mathbb{C})$ to $H^{2}(X, \mathbb{C})_{\text {prim. }}$. For $\gamma \in H^{2}(X, \mathbb{C})$, denote by $\gamma_{\text {prim }}$ the image of $\gamma$ under this morphism. Since the very ample line bundle $H_{X}$ remains of type $(1,1)$ in the family $\mathcal{X}$, we can therefore conclude that $\gamma \in H^{1,1}(X)$ remains of type $(1,1)$ if and only if $\gamma_{\text {prim }}$ remains of type $(1,1)$. In particular, $\operatorname{NL}(\gamma)=\operatorname{NL}\left(\gamma_{\text {prim }}\right)$.

Definition 2.5. We now discuss the tangent space to the Hodge locus, $\operatorname{NL}(\gamma)$. We know that the tangent space to $U$ at $X, T_{X} U$ is isomorphic to $H^{0}\left(\mathcal{N}_{X \mid \mathbb{P}^{3}}\right)$. This is because $U$ is an open subscheme of the Hilbert scheme $H_{Q_{d}}$, the tangent space of which at the point $X$ is simply $H^{0}\left(\mathcal{N}_{X \mid \mathbb{P}^{3}}\right)$. Given the variation of Hodge structure above, we have (by Griffith's transversality) the differential map:

$$
\bar{\nabla}: H^{1,1}(X) \rightarrow \operatorname{Hom}\left(T_{X} U, H^{2}\left(X, \mathcal{O}_{X}\right)\right)
$$


induced by the Gauss-Manin connection. Given $\gamma \in H^{1,1}(X)$ this induces a morphism, denoted $\bar{\nabla}(\gamma)$ from $T_{X} U$ to $H^{2}\left(\mathcal{O}_{X}\right)$. The tangent space at $X$ to $\mathrm{NL}(\gamma)$ is then defined to be $\operatorname{ker}(\bar{\nabla}(\gamma))$.

2.6. The boundary map

$$
\rho: H^{0}\left(\mathcal{N}_{X \mid \mathbb{P}^{3}}\right) \rightarrow H^{1}\left(\mathcal{T}_{X}\right)
$$

arising from the long exact sequence associated to the short exact sequence:

$$
\left.0 \rightarrow \mathcal{T}_{X} \rightarrow \mathcal{T}_{\mathbb{P}^{3}}\right|_{X} \rightarrow \mathcal{N}_{X \mid \mathbb{P}^{3}} \rightarrow 0
$$

is called the Kodaira-Spencer map. The morphism $\bar{\nabla}(\gamma)$ is related to the Kodaira-Spencer map as we will see below.

2.7. Note that there exists a natural cup product morphism,

$$
H^{1}\left(X, \mathcal{T}_{X}\right) \otimes H^{1}\left(X, \Omega_{X}^{1}\right) \stackrel{\bigcup}{\longrightarrow} H^{2}\left(X, \mathcal{O}_{X}\right)
$$

For $\gamma \in H^{1}\left(\Omega_{X}^{1}\right)$ this induces a morphism, denoted $\bigcup \gamma$, from $H^{1}\left(\mathcal{T}_{X}\right)$ to $H^{2}\left(\mathcal{O}_{X}\right)$. We then have the following result in Hodge theory (see Voi02, Theorem 10.21]):

Lemma 2.8. The differential map $\bar{\nabla}(\gamma)$ conincides with the following:

$$
T_{X} U \cong H^{0}\left(\mathcal{N}_{X \mid \mathbb{P}^{3}}\right) \stackrel{\rho}{\rightarrow} H^{1}\left(\mathcal{T}_{X}\right) \stackrel{\bigcup \gamma}{\longrightarrow} H^{2}\left(\mathcal{O}_{X}\right)
$$

\section{$3 \quad$ Hodge locus and Hilbert flag schemes}

3.1. In this section we define what is a semi-regular map. We then briefly study Hodge locus for a family of smooth projective surfaces in $\mathbb{P}^{3}$ and show how it is related to certain Hilbert flag schemes. More specifically, we shall study the Hodge locus corresponding to certain effective algebraic cycles which will be semi-regular. For such classes we will see that the Hodge locus "coincides" with a component of a flag Hilbert scheme. We elaborate on the details in this section. 


\subsection{Semi-regularity map and tangent space to Hodge locus}

3.2. We start with the definition of a semi-regular curve. Let $X$ be a surface and $C \subset X$, a curve in $X$. Since $X$ is smooth, $C$ is local complete intersection in $X$. This gives rise to the short exact sequence:

$$
0 \rightarrow \mathcal{O}_{X}(-C) \rightarrow \mathcal{O}_{X} \rightarrow i_{*} \mathcal{O}_{C} \rightarrow 0
$$

where $i$ is the natural inclusion morphism from $C$ into $X$. Note that, $\mathcal{O}_{X}(C)$ is locally free $\mathcal{O}_{X}$-module, hence flat. Therefore, tensoring this short exact sequence by $\mathcal{O}_{X}(C)$ we get

$$
0 \rightarrow \mathcal{O}_{X} \rightarrow \mathcal{O}_{X}(C) \rightarrow \mathcal{N}_{C \mid X} \rightarrow 0
$$

is exact, where $\mathcal{N}_{C \mid X}$ is the normal sheaf $\mathcal{H o m}_{X}\left(\mathcal{O}_{X}(-C), i_{*} \mathcal{O}_{C}\right)$ which is isomorphic to the sheaf $i_{*} \mathcal{O}_{C} \otimes_{\mathcal{O}_{X}} \mathcal{O}_{X}(C)$ (see [Har77, Ex. II.5.1(b)]). The semi-regularity map is the morphism

$$
\pi: H^{1}\left(\mathcal{N}_{C \mid X}\right) \rightarrow H^{2}\left(\mathcal{O}_{X}\right)
$$

which arises from the long exact sequence associated to the short exact sequence (11). We say that $C$ is semi-regular if $\pi$ is injective.

3.3. The Lefschetz hyperplane section theorem implies that $H^{1}\left(\mathcal{O}_{X}\right)=0$. Then, the long exact sequence associated to (1) contains the following segment:

$$
0 \rightarrow H^{1}\left(\mathcal{O}_{X}(C)\right) \rightarrow H^{1}\left(\mathcal{N}_{C \mid X}\right) \stackrel{\pi}{\rightarrow} H^{2}\left(\mathcal{O}_{X}\right)
$$

So, $H^{1}\left(\mathcal{O}_{X}(C)\right)=0$ is equivalent to $\pi$ being injective, hence $C$ being semi-regular. We now prove a result that would help us determine when a curve is semi-regular.

Lemma 3.4. Let $C$ be a connected reduced curve and $d \geq \operatorname{deg}(C)+4$ then $h^{1}\left(\mathcal{O}_{X}(C)\right)=0$. In particular, $C$ is semi-regular.

Proof. Since $X$ is a hypersurface in $\mathbb{P}^{3}$ of degree $d, \mathcal{I}_{X} \cong \mathcal{O}_{\mathbb{P}^{3}}(-d)$. Consider the short exact sequence:

$$
0 \rightarrow \mathcal{I}_{X} \rightarrow \mathcal{I}_{C} \rightarrow \mathcal{O}_{X}(-C) \rightarrow 0
$$


Tensoring this by $\mathcal{O}_{\mathbb{P}^{3}}(k)$, we get the following terms in the associated long exact sequence:

$$
\ldots \rightarrow H^{1}\left(\mathcal{I}_{C}(k)\right) \rightarrow H^{1}\left(\mathcal{O}_{X}(-C)(k)\right) \rightarrow H^{2}\left(\mathcal{I}_{X}(k)\right) \rightarrow \ldots
$$

Now, $H^{2}\left(\mathcal{O}_{\mathbb{P}^{3}}(k-d)\right)=0$ (see Har77, Theorem 5.1]) and $\mathcal{I}_{C}$ is $\operatorname{deg}(C)$-regular (see Gia06, Main Theorem]). So, $H^{1}\left(\mathcal{I}_{C}(k)\right)=0$ for $k \geq \operatorname{deg}(C)$. This implies $H^{1}\left(\mathcal{O}_{X}(-C)(k)\right)=0$ for $k \geq \operatorname{deg}(C)$. By Serre duality, $0=H^{1}\left(\mathcal{O}_{X}(-C)(d-4)\right) \cong H^{1}\left(\mathcal{O}_{X}(C)\right)$. So, $C$ is semi-regular.

3.5. Let $X$ be a surface and $C \subset X$ be a curve. We now do a computation to show that for $d \geq \operatorname{deg}(C)+4, \operatorname{dim}|C|=0$, where $|C|$ is the linear system of $C$ in $X$.

Lemma 3.6. Let $d \geq 5$ and $C$ be an effective divisor on a smooth degree $d$ surface $X$ of the form $\sum_{i} a_{i} C_{i}$, where $C_{i}$ are integral curves with $\operatorname{deg}(C)+4 \leq d$. Then, $h^{0}\left(\mathcal{N}_{C \mid X}\right)=0$. In particular, $\operatorname{dim}|C|=0$, where $|C|$ is the linear system associated to $C$.

Proof. Let $C=\sum_{i} a_{i} C_{i}$ with $C_{i}$ integral. Then,

$$
\operatorname{deg}\left(\left.\left(\left.\mathcal{O}_{X}(C)\right|_{C} \otimes \mathcal{O}_{C}\right)\right|_{C_{i}}\right)=a_{i} C_{i}^{2}+\sum_{j \neq i} a_{j} C_{i} \cdot C_{j}
$$

Denote by $e_{i}:=\operatorname{deg}\left(C_{i}\right)$. Using the adjunction formula and the fact that $K_{X} \cong \mathcal{O}_{X}(d-4)$, we have that

$$
\begin{aligned}
\operatorname{deg}\left(\left.\left(\left.\mathcal{O}_{X}(C)\right|_{C} \otimes \mathcal{O}_{C}\right)\right|_{C_{i}}\right) & =2 a_{i} \rho_{a}\left(C_{i}\right)-2 a_{i}-(d-4) a_{i} e_{i}+\sum_{j \neq i} a_{j} C_{i} \cdot C_{j} \\
& \leq a_{i}\left(e_{i}^{2}-(d-1) e_{i}\right)+\sum_{j \neq i} a_{j} C_{i} C_{j} \\
& \leq a_{i}\left(e_{i}^{2}-3 e_{i}-e_{i} \sum_{j} a_{j} e_{j}\right)+\sum_{j \neq i} a_{j} e_{i} e_{j} .
\end{aligned}
$$

The first inequality follows from the bound on the genus of a curve in $\mathbb{P}^{3}$ in terms of its degree (see [Har77, Example 6.4.2]). The second inequality follows from the facts that $d \geq \operatorname{deg}(C)+4$ and $C_{i} . C_{j} \leq e_{i} e_{j}$. It then follows directly that $\operatorname{deg}\left(\left.\left(\left.\mathcal{O}_{X}(C)\right|_{C} \otimes \mathcal{O}_{C}\right)\right|_{C_{i}}\right)<0$. This implies that $h^{0}\left(C_{i},\left.\left(\left.\mathcal{O}_{X}(C)\right|_{C} \otimes \mathcal{O}_{C}\right)\right|_{C_{i}}\right)=0$ for all $i$. So, $h^{0}\left(\mathcal{N}_{C \mid X}\right)=h^{0}\left(C,\left.\mathcal{O}_{X}(C)\right|_{C} \otimes \mathcal{O}_{C}\right)=0$.

Since $h^{1}\left(\mathcal{O}_{X}\right)=0$ (by Lefschetz hyperplane section Theorem) and $h^{0}\left(\mathcal{O}_{X}\right)=1$, using the 
long exact sequence associated to the short exact sequence

$$
\left.0 \rightarrow \mathcal{O}_{X} \rightarrow \mathcal{O}_{X}(C) \rightarrow \mathcal{O}_{X}(C)\right|_{C} \otimes \mathcal{O}_{C} \rightarrow 0
$$

we get that $h^{0}\left(\mathcal{O}_{X}(C)\right)=1$. Since $|C|=\mathbb{P}\left(H^{0}\left(\mathcal{O}_{X}(C)\right)\right)$, the lemma follows.

\subsection{Flag Hilbert scheme and Hodge locus}

3.7. In this section we introduce the basic definitions of flag Hilbert schemes. See [Ser06, §4] for further details. We then prove the main result of this section which relates Hodge locus to Hilbert schemes.

3.8. Given an $m$-tuple of polynomials $\mathcal{P}(t)=\left(P_{1}(t), P_{2}(t), \ldots, P_{m}(t)\right)$, we define the contravariant functor, called the Hilbert flag functor relative to $\mathcal{P}(t)$,

$$
\begin{gathered}
F H_{\mathcal{P}(t)}:(\text { schemes }) \rightarrow \text { sets } \\
S \mapsto\left\{\left(X_{1}, X_{2}, \ldots, X_{m}\right) \mid X_{1} \subset X_{2} \subset \ldots \subset \mathbb{P}_{S}^{3}\right\}
\end{gathered}
$$

such that the Hilbert polynomial of $X_{i}$ is $P_{i}(t)$ and $X_{i}$ is an $S$-closed subscheme of $X_{i+1}$. We call such an $m$-tuple a flag relative to $\mathcal{P}(t)$.

3.9. The functor $F H_{\mathcal{P}(t)}$ is representable by a projective scheme, $H_{\mathcal{P}(t)}$ which parametrizes all such flags relative to $\mathcal{P}(t)$. We call this the Hilbert flag scheme.

Theorem 3.10. Let $X$ be a surface, $C$ be a semi-regular curve in $X$ and $\gamma \in H^{1,1}(X, \mathbb{Z})$ be the class of the curve $C$. For any irreducible component $L^{\prime}$ of $\overline{\mathrm{NL}(\gamma)}$ (the closure is taken in the Zariski topology on $U_{d}$ ) there exists an irreducible component $H^{\prime}$ of $H_{P, Q_{d}}$ containing the pair $(C, X)$ such that the associated reduced scheme $\operatorname{pr}_{2}\left(H^{\prime}\right)_{\text {red }}$ coincides with $L_{\text {red }}^{\prime}$, where $\operatorname{pr}_{2}$ is the second projection map from $H_{P, Q_{d}}$ to $H_{Q_{d}}$. Furthermore, if $d \geq \operatorname{deg}(C)+4$ then such $H^{\prime}$ is uniquely determined by $L^{\prime}$.

Proof. The first part of the theorem follows directly from [Blo72, Theorem 7.1].

Furthermore, Lemma 3.6 implies that $\operatorname{dim}|C|=0$. So, given an irreducible component, say $L^{\prime}$ of $\overline{\mathrm{NL}(\gamma)}$ such that $X$ is a general element, there exists an unique irreducible component $H^{\prime}$ 
of $H_{P, Q_{d}}$ containing the pair $(C, X)$ such that $\operatorname{pr}_{2}\left(H^{\prime}\right)_{\text {red }}$ coincides with $L_{\text {red }}^{\prime}$. This proves the rest of the theorem.

\section{Variation of lattices}

4.1. In this section we give a formula to compute the dimension of an irreducible component of a Hodge locus (see Proposition 4.6). This result will be particularly useful to prove the asymptotic case of a Griffiths-Harris conjecture, which we see in the next section.

4.2. Let $X$ be a surface of degree $d$. An augmented lattice $\Lambda_{X}$ on $X$ of rank $r$, is a rank $r \mathbb{Z}$-submodule $\Lambda_{X} \subset H^{2}(X, \mathbb{Z})$ generated by the class of the very ample line bundle $H_{X}$ (as in 2.3) and cohomology classes of $r-1$ reduced curves, say $C_{1}, \ldots, C_{r-1}$ such that $\Lambda_{X}$ is saturated in the sense that for all $\lambda \in \Lambda_{X}, c \in \mathbb{Q}$ if $c \lambda \in H^{2}(X, \mathbb{Z})$ then $c \lambda \in \Lambda_{X}$. For such $\Lambda_{X}$, we say that $C_{i}$ for $i=1, \ldots, r-1$ generate $\Lambda_{X}$. We say that $\Lambda_{X}$ is prime if $C_{1}, \ldots, C_{r-1}$ are integral.

4.3. Let $\Lambda_{X}$ be as in 4.2 . We can define

$$
\mathrm{NL}\left(\Lambda_{X}\right):=\left\{G \in U \mid \bar{\gamma}_{G}=0, \text { for all } \gamma \in \Lambda_{X}\right\}
$$

For a surface $X$ and reduced curve $C \subset X$ such that the cohomology class $[C]$ of $C$ is not a $\mathbb{Q}$-multiple of $c_{1}\left(H_{X}\right)$, denote by $\Lambda_{X}^{0}$ the rank $2 \mathbb{Z}$-module generated by $[C]$ and $c_{1}\left(H_{X}\right)$, where $c_{1}$ is the first Chern class map. Since a very ample line bundle remains of type $(1,1)$ is the family $\mathcal{X}, \operatorname{NL}\left(\Lambda_{X}^{0}\right)$ coincides with $\mathrm{NL}([C])$. More generally, for a rank $r$ augmented lattice $\Lambda_{X}$ generated by $C_{1}, \ldots, C_{r-1}$ we have, $\mathrm{NL}\left(\Lambda_{X}\right)$ is isomorphic to the fiber product $\mathrm{NL}\left(\left[C_{1}\right]\right) \times_{H_{Q_{d}}}$ $\ldots \times{ }_{H_{Q_{d}}} \mathrm{NL}\left(\left[C_{r-1}\right]\right)$.

4.4. Let $\Lambda_{X}$ be as before of rank 2 , generated by a reduced curve, say $C$. Let $P$ be the Hilbert polynomial of $C$. Assume $d \geq \operatorname{deg}(C)+4$. Using Theorem 3.10 we can conclude that for general $X^{\prime} \in \mathrm{NL}\left(\Lambda_{X}\right)$ there exists a curve $C^{\prime} \subset X^{\prime}$ such that $\overline{\mathrm{NL}\left(\left[C^{\prime}\right]\right)}$ is an irreducible component of $\overline{\mathrm{NL}\left(\Lambda_{X}\right)}$ and $C^{\prime}$ deforms to $C$, i.e., $C^{\prime}$ has the same Hilbert polynomial $P$. Denote by $\Lambda_{X^{\prime}}$ the augmented lattice on $X^{\prime}$ of rank 2 generated by $C^{\prime}$. Theorem 3.10 again implies that there exists 
an unique irreducible component, denoted $H_{\Lambda_{X^{\prime}}}$, of $H_{P, Q_{d}}$ containing the pair $\left(C^{\prime}, X^{\prime}\right)$ such that $\operatorname{pr}_{2}\left(H_{\Lambda_{X^{\prime}}}\right)$ is isomorphic to $\overline{\mathrm{NL}\left(\Lambda_{X^{\prime}}\right)}$. Denote by $L_{\Lambda_{X^{\prime}}}:=\operatorname{pr}_{1}\left(H_{\Lambda_{X^{\prime}}}\right)$. From now on, we will always assume that $\overline{\mathrm{NL}\left(\Lambda_{X}\right)}$ is irreducible, which is equivalent to $X$ being general in $\operatorname{NL}\left(\Lambda_{X}\right)$, in particular, away from the points of intersection of any two irreducible components of $\overline{\operatorname{NL}\left(\Lambda_{X}\right)}$.

4.5. Suppose now that $\Lambda_{X}$ is of rank $r$ generated by $C_{1}, \ldots, C_{r-1}$. Let $P_{1}, \ldots, P_{r-1}$ be the Hilbert polynomials of $C_{1}, \ldots, C_{r-1}$, respectively. Consider the natural morphism

$$
p: H_{P_{1}, Q_{d}} \times_{H_{Q_{d}}} \ldots \times_{H_{Q_{d}}} H_{P_{r-1}, Q_{d}} \rightarrow H_{Q_{d}} .
$$

Assume $d \geq \sum_{i=1}^{r-1} \operatorname{deg}\left(C_{i}\right)+4$. Using Theorem 3.10, we can conclude that for every irreducible component $L^{\prime}$ of $\overline{\mathrm{NL}\left(\Lambda_{X}\right)}$ there exists an unique irreducible component, say $H$ of $H_{P_{1}, Q_{d}} \times_{H_{Q_{d}}}$ $\ldots \times{ }_{H_{Q_{d}}} H_{P_{r-1}, Q_{d}}$ containing $\left(C_{1}, X\right) \times \ldots \times\left(C_{r-1}, X\right)$ such that $p(H)$ coincides with $L^{\prime}$. Similarly as in 4.4 by taking $X$ general in $\operatorname{NL}\left(\Lambda_{X}\right)$ we can ensure that $\overline{\operatorname{NL}\left(\Lambda_{X}\right)}$ is irreducible. Denote by $H_{\Lambda_{X}}$ the irreducible component of $H_{P_{1}, Q_{d}} \times_{H_{Q_{d}}} \ldots \times_{H_{Q_{d}}} H_{P_{r}, Q_{d}}$ such that $p\left(H_{\Lambda_{X}}\right)$ coincides with $\overline{\operatorname{NL}\left(\Lambda_{X}\right)}$. Denote by $L_{\Lambda_{X}}:=\operatorname{pr}\left(H_{\Lambda_{X}}\right)$, where pr is the natural projection map from $H_{P_{1}, Q_{d}} \times_{H_{Q_{d}}}$ $\ldots \times{ }_{H_{Q_{d}}} H_{P_{r-1}, Q_{d}}$ to $H_{P_{1}} \times \ldots \times H_{P_{r-1}}$.

Proposition 4.6. Let $r \geq 3, X$ be a surface of degree $d$ and $\Lambda_{X}$ be an augmented lattice of rank $r+1$ generated by $r$ reduced curves $C_{1}, \ldots, C_{r}$. Assume that $\sum_{i=1}^{r} \operatorname{deg}\left(C_{i}\right)+4 \leq d$. Then, the dimension of $\overline{\mathrm{NL}\left(\Lambda_{X}\right)}$ is given by the following formula:

$$
\operatorname{codim} \overline{\mathrm{NL}\left(\Lambda_{X}\right)}=\operatorname{codim} I_{d}(C)-\operatorname{dim} L_{\Lambda_{X}}
$$

where $C=C_{1}^{\prime} \bigcup \ldots \cup C_{r}^{\prime}$ for a general $r$-tuple $\left(C_{1}^{\prime}, \ldots, C_{r}^{\prime}\right)$ in $L_{\Lambda_{X}}$ and $I_{d}(C)$ is the degree $d$ graded piece in the ideal $I(C)$ of $C$.

Proof. Consider the diagram,

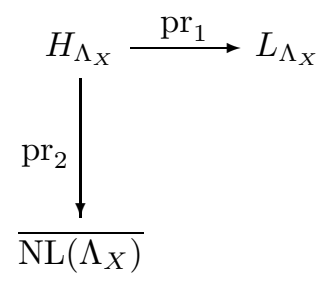

Denote by $P_{i}$ the Hilbert polynomial of curves $C_{i}$, respectively. Recall, $L_{\Lambda_{X}}$ is contained in 
$H_{P_{1}} \times \ldots \times H_{P_{r}}$. For an $r$-tuple $\left(C_{1}, \ldots, C_{r}\right) \in L_{\Lambda_{X}}$, the fiber of $\operatorname{pr}_{1}$ parametrizes the space of smooth degree $d$ surfaces containing $C=C_{1} \bigcup \ldots \cup C_{r}$, which is an open subscheme in $\mathbb{P}\left(I_{d}(C)\right)$. Since $I_{d}(C)$ is irreducible, the dimension of the generic fiber of $\operatorname{pr}_{1}$ is equal to $\operatorname{dim} I_{d}(C)-1$, where $\left(C_{1}, \ldots, C_{r}\right) \in L_{\Lambda_{X}}$ is a general element. The fiber of $\operatorname{pr}_{2}$ over $\operatorname{pr}_{2}\left(\left(C_{1}, \ldots, C_{r}, X\right)\right)$ is isomorphic to $\left|C_{1}\right| \times \ldots \times\left|C_{r}\right|$. But, Lemma 3.6 implies $\operatorname{dim}\left|C_{i}\right|=0$ for $i=1, \ldots, r$. So, the dimension of the generic fiber of $\mathrm{pr}_{2}$ is zero. Then,

$$
\operatorname{dim} H_{\Lambda_{X}}=\operatorname{dim} L_{\Lambda_{X}}+\operatorname{dim} I_{d}(C)-1=\operatorname{dim} \mathrm{NL}\left(\Lambda_{X}\right) .
$$

So, $\operatorname{codim} \overline{\mathrm{NL}\left(\Lambda_{X}\right)}=\operatorname{dim} \mathbb{P}\left(H^{0}\left(\mathcal{O}_{\mathbb{P}^{3}}(d)\right)\right)-\operatorname{dim} \overline{\mathrm{NL}\left(\Lambda_{X}\right)}=h^{0}\left(\mathcal{O}_{\mathbb{P}^{3}}(d)\right)-\operatorname{dim} I_{d}(C)-\operatorname{dim} L_{\Lambda_{X}}$.

This finishes the proof of the proposition.

\section{A Griffiths-Harris conjecture}

5.1. We now come to the final section of the article, where we prove an asymptotic case of a Griffiths-Harris conjecture. Recall, a Griffiths-Harris conjecture states in [GH83] that:

For $3 \leq r \leq d$, the codimension of an irreducible component of $\mathrm{NL}_{r, d}$ is at least equal to

$$
(r-1)(d-3)-\left(\begin{array}{c}
r-3 \\
2
\end{array}\right)
$$

Furthermore, there exists a component of $\mathrm{NL}_{r, d}$ of this codimension parametrizing the space of surfaces containing $r-1$ lines on the same plane.

Notation 5.2. We will denote by $\mathcal{N}_{d}(r)$ the number,

$$
(d-3)(r-1)-\left(\begin{array}{c}
r-3 \\
2
\end{array}\right)
$$

We now recall a result in Noether-Lefschetz locus due to Otwinowska which will help us characterize the irreducible components of $\mathrm{NL}_{r, d}$ with codimension less than or equal to $\mathcal{N}_{d}(r)$.

Theorem 5.3 ([Otw04, Theorem 1]). Let $\gamma$ be an augmented lattice of rank 2 on a degree $d$ surface. There exists $C \in \mathbb{R}_{+}^{*}$ depending only on $r$ such that for $d \geq C(r-1)^{8}$ if $\operatorname{codim} \mathrm{NL}(\gamma) \leq$ 
$(r-1) d$ then $\gamma_{\text {prim }}=\sum_{i=1}^{t} a_{i}\left[C_{i}\right]_{\text {prim }}$, where $a_{i} \in \mathbb{Q}^{*}, C_{i}$ are reduced curves and $\operatorname{deg}\left(C_{i}\right) \leq(r-1)$ for $i=1, \ldots, t$ for some positive integer $t$.

5.4. Throughout this section we denote by $r$ an integer greater than or equal to 3 and for a fixed $r$, denote by $d$, an integer as mentioned in Theorem 5.3. We will assume that $d$ is at least $r^{3}$ which will be used only in a computation in Lemma 5.9, The other results do not have any restriction on $d$ in terms of $r$.

Proposition 5.5. Let $L$ be an irreducible component of $\mathrm{NL}_{r, d}$. Then $L$ is locally homeomorphic to $\operatorname{NL}(\Lambda)$ for some prime augmented lattice $\Lambda$ of rank at least $r$ on a surface $X \in L$, general.

Proof. Let $L \subset \mathrm{NL}_{r, d}$ be an irreducible component. Let $X$ be a general element in $L$. This implies that for the Picard lattice $\Lambda:=\operatorname{NS}(X), \operatorname{NL}(\Lambda)_{\text {red }}$ is an open subscheme of $L_{\text {red }}$. We can assume that $\Lambda$ is a prime lattice. Since $X$ is an element in $\mathrm{NL}_{r, d}$, the rank of $\Lambda$ is greater than or equal to $r$.

Proposition 5.6. If $\Lambda$ is an augmented prime lattice of rank $t$ on some degree $d$ surface and $\operatorname{codim} \overline{\mathrm{NL}(\Lambda)} \leq(r-1) d$. Then there exists a prime lattice $\Lambda^{\prime}$ of rank greater than or equal to $t$ generated by classes of curves of degree less than or equal to $r-1$, such that $C_{i}$ deforms along $\mathrm{NL}\left(\Lambda^{\prime}\right)$ and $\overline{\mathrm{NL}(\Lambda)}_{\mathrm{red}}={\overline{\mathrm{NL}\left(\Lambda^{\prime}\right)_{\mathrm{red}}}}_{\text {. }}$

Proof. Let $X \in \mathrm{NL}(\Lambda)$. There exists a maximal lattice $\Lambda^{\prime} \subset H^{2}(X, \mathbb{Z})$ such that $\Lambda^{\prime}$ remains of type $(1,1)$ in $\mathrm{NL}(\Lambda)$ i.e., $\mathrm{NL}(\Lambda)_{\text {red }}=\mathrm{NL}\left(\Lambda^{\prime}\right)_{\text {red }}$. Now, there exists a surface $X^{\prime} \in \mathrm{NL}\left(\Lambda^{\prime}\right)$ such that the Néron-Severi group $\operatorname{NS}\left(X^{\prime}\right)$ is the translate (under deformation from $X$ to $X^{\prime}$ ) of $\Lambda^{\prime}$ in $H^{2}\left(X^{\prime}, \mathbb{Z}\right)$ which we again denote by $\Lambda^{\prime}$ for convinience of notation. Then Theorem 5.3 implies that any $\gamma \in \Lambda^{\prime}$ is of the form $\sum_{i} a_{i}\left[C_{i}\right]+b H_{X}$ with $\operatorname{deg}\left(C_{i}\right) \leq r-1$. So, $\Lambda^{\prime}$ can be generated by classes of curves of degree at most $r-1$ and the class of the very ample line bundle $H_{X}$. Now, the class of $\left[C_{i}\right]$ remains of type $(1,1)$ along $\mathrm{NL}\left(\Lambda^{\prime}\right)$. From Lemma 3.4 it follows that $C_{i}$ is semi-regular. Then, Blo72, Theorem 7.1] implies that the class of $\left[C_{i}\right]$ remains effective along $\mathrm{NL}\left(\Lambda^{\prime}\right)$. This proves the proposition.

5.7. We now recall a result due to Eisenbud and Harris which we use in the next lemma. Let $P$ be a Hilbert polynomial of a curve in $\mathbb{P}^{3}$ of degree $e$ and $L$ be an irreducible component of $H_{P}$. The corollary after [EH92, Theorem 1] tells us that, 
Theorem 5.8 ([EH92]). For $e>1$, the dimension of $L$ is less than or equal to $3+e(e+3) / 2$.

Lemma 5.9. Let $\Lambda$ be a prime augmented lattice of rank $t+1$ on a degree $d$ surface, generated by irreducible curves $C_{i}$ for $i=1, \ldots, t$ for some positive integer $t$ and $\operatorname{deg}\left(C_{i}\right) \leq r-1$. Suppose $\operatorname{codim} \mathrm{NL}(\Lambda) \leq(r-1) d$. Then, $\sum_{i=1}^{t} \operatorname{deg}\left(C_{i}\right) \leq(r-1)$.

Proof. We prove this by induction on $t$. This is trivially true for $t=1$. Suppose this is true for all $t \leq m$.

Assume this is not true for $t=m+1$. In other words, there exists a prime lattice $\Lambda$ minimally generated by $m+1$ curves such that $\sum_{i} \operatorname{deg}\left(C_{i}\right)>r-1$. This implies (after rearranging the indices if necessary) there exists an integer $0<t^{\prime} \leq m+1$ such that $C_{1}, \ldots, C_{t^{\prime}}$ satisfies $\sum_{i=1}^{t^{\prime}} \operatorname{deg}\left(C_{i}\right)>(r-1)$ and $\sum_{i=1}^{t^{\prime}-1} \operatorname{deg}\left(C_{i}\right) \leq(r-1)$. Then, $\sum_{i=1}^{t^{\prime}} \operatorname{deg}\left(C_{i}\right) \leq 2(r-1)$. Denote by $P$ the Hilbert polynomial of the curve $C_{1} \cup \ldots \cup C_{t^{\prime}}$. We replace $e$ by $2(r-1)$ in Theorem 5.8 and conclude that the dimension of the Hilbert scheme $H_{P}$ is less than or equal to $3+(r-$ 1) $(2 r+1)$. Using Proposition 4.6, the codimension of $\mathrm{NL}\left(\left[C_{1}+\ldots+C_{t^{\prime}}\right]\right)$ is greater than or equal to $\operatorname{codim} I_{d}\left(C_{1} \cup \ldots \cup C_{t^{\prime}}\right)-\operatorname{dim} H_{P}$. Since $r-1<\operatorname{deg}\left(C_{1}+\ldots+C_{t^{\prime}}\right) \leq 2(r-1)$ and $d \geq r^{3}$, we get the following inequality using the upper bound on the arithmetic genus of a curve of degree less than or equal to $2(r-1)$ :

$$
\begin{aligned}
\operatorname{codim} \mathrm{NL}\left(\left[C_{1}+\ldots+C_{t^{\prime}}\right]\right) & \geq \operatorname{codim} I_{d}\left(C_{1}+\ldots C_{t^{\prime}}\right)-\operatorname{dim} H_{P} \\
& \geq(r d-(2 r-3)(2 r-4) / 2+1)-(3+(r-1)(2 r+1)) \\
& =(r-1) d+(d-(2 r-3)(2 r-4) / 2-3-(r-1)(2 r+1)+1) \\
& >(r-1) d
\end{aligned}
$$

contradicting the assumption.

Proposition 5.10. Let $\Lambda$ be an augmented lattice of rank $r$ contained in a degree $d$ surface, generated by $l_{i}$ for $i=1, \ldots, r-1$, where $l_{i}$ are lines for all $i$. Suppose $r \geq 3$. Then, $\operatorname{codim} \operatorname{NL}(\Lambda) \geq$ $\mathcal{N}_{d}(r)$. Furthermore, if $l_{i}$ are on the same plane we have an equality. 
Proof. We prove this by induction. Using Proposition 4.6 ,

$$
\operatorname{codim} \mathrm{NL}(\Lambda)=\operatorname{codim} I_{d}\left(\bigcup_{i=1}^{r-1} l_{i}\right)-\operatorname{dim} L_{\Lambda} .
$$

If $r=3$, codim $\mathrm{NL}(\Lambda)=2 d-6=\mathcal{N}_{d}(3)$. Assume the result holds true for all $r \leq m$ for some integer $m \geq 4$. We now prove for $r=m+1$. Denote by $\Lambda^{\prime}$ the lattice generated by $l_{i}$ for $i=1, \ldots, r-2$ and by $C$ the curve $\bigcup_{i=1}^{r-2} l_{i}$. Let $t:=\operatorname{dim} L_{\Lambda}-\operatorname{dim} L_{\Lambda^{\prime}}$. Note that, $t \leq 4$.

Note that $l_{r-1} . C \leq r-2$. Denote by $\epsilon:=r-2-l_{r-1} . C$. Comparing with the list of values of $t$, we see

1. If $\epsilon=0$ then $l_{r-1} . C=r-2$ and $t \leq 2$. In particular, for a fixed curve $C$ there is a 1-1 correspondence between the set of choices of $l_{r-1}$ intersecting $C$ in $r-2$ points and the set of planes $P$ interesting $C$ at $r-2$ distinct collinear points. If for a generic choice of $P$, $P \cap C$ are $r-2$ distinct collinear points then all lines in $C$ should lie on the same plane. In that case, $l_{r-1}$ intersect $C$ at $r-2$ points if and only if $l_{r-1}$ is on the same plane as $C$, hence $t=2$ (dimension of the space of lines in $\mathbb{P}^{2}$ ). If this is not the case i.e., a generic choice of $P$ does not intersect $C$ in $r-2$ distinct collinear points, then $t \leq 2$.

2. $\epsilon=r-2$ and $t=4$ if $l_{r-1}$ does not intersect $C$.

3. $0<\epsilon<r-2$ and $t \leq 3$ otherwise.

Now,

$$
\begin{aligned}
\operatorname{codim} \mathrm{NL}(\Lambda) & =\operatorname{codim} I_{d}\left(C \bigcup l_{r-1}\right)-\operatorname{dim} L_{\Lambda} \\
& =\left(\operatorname{codim} I_{d}(C)+\operatorname{codim} I_{d}\left(l_{r-1}\right)-l_{r-1} \cdot C\right)-\operatorname{dim} L_{\Lambda} \\
& =\left(\operatorname{codim} I_{d}(C)-\operatorname{dim} L_{\Lambda^{\prime}}\right)+\left(\operatorname{codim} I_{d}\left(l_{r-1}\right)-t\right)-(r-2-\epsilon) .
\end{aligned}
$$

Writing $t=4-(4-t)$ and using the induction step, we see that the right hand side of this 
equation is greater than or equal to

$$
\begin{array}{r}
\left((r-2)(d-3)-\left(\begin{array}{c}
r-4 \\
2
\end{array}\right)\right)+(d-3)-(r-2-\epsilon)+(4-t) \\
=\left((r-2)(d-3)-\left(\begin{array}{c}
r-3 \\
2
\end{array}\right)\right)+\epsilon-t+2
\end{array}
$$

Note that $\epsilon-t+2 \geq 0$ from the three cases considered above. Substituting this inequality gives us codim $\mathrm{NL}(\Lambda) \geq \mathcal{N}_{d}(r)$.

Theorem 5.11. Let $L$ be an irreducible component of $\mathrm{NL}_{r, d}$. Then, $\operatorname{codim} L \geq(r-1)(d-$

$3)-\left(\begin{array}{c}r-3 \\ 2\end{array}\right)$. Furthermore, there exists a component $L$ of $\mathrm{NL}_{r, d}$ of this codimension parametrizing surfaces containing $r-1$ coplanar lines.

Proof. It suffices to prove $L$ is locally of the form $\operatorname{NL}(\Lambda)$ for $\Lambda$ a rank $r-1$ prime lattice generated by $\left[l_{i}\right]$ for $i=1, \ldots, r$, where $l_{i}$ are lines on the same plane. Using Proposition [5.5 $L$ is of the form $\mathrm{NL}(\Lambda)$ for a rank $r-1$ prime lattice $\Lambda$. Using Proposition 5.6 we can assume that there exists a prime lattice $\Lambda^{\prime}$ of rank $t$ greater than or equal to $r-1$ generated by classes of curves, say $C_{i}^{\prime}$ for $i=1, \ldots, t$ of degree less than or equal to $r-1$ such that $\mathrm{NL}(\Lambda)=\mathrm{NL}\left(\Lambda^{\prime}\right)$. Lemma 5.9 implies that $t=r-1$ and $\operatorname{deg}\left(C_{i}^{\prime}\right)=1$ for all $i=1, \ldots, r-1$. Finally, the theorem follows from Proposition 5.10

\section{References}

[Blo72] S. Bloch. Semi-regularity and de-Rham cohomology. Inventiones Math., 17:51-66, 1972.

[CHM88] C. Ciliberto, J. Harris, and R. Miranda. General components of the Noether-Lefschetz locus and their density in the space of all surfaces. Mathematische Annalen, 282(4):667$680,1988$.

[EH92] D. Eisenbud and J. Harris. The dimension of the Chow variety of curves. Compositio Mathematica, 83:291-310, 1992. 
[GH83] P. Griffiths and J. Harris. Infinitesimal variations of hodge structure (ii): an infinitesimal invariant of hodge classes. Composition Mathematica, 50(2-3):207-265, 1983.

[Gia06] D. Giaimo. On the castelnuovo-mumford regularity of connected curves. Transactions of the American Mathematical society, 358(1):267-284, 2006.

[Gre89] M. Green. Components of maximal dimension in the Noether-Lefschetz locus. $J$. Differential Geometry, 29:295-302, 1989.

[Har77] R. Hartshorne. Algebraic Geometry. Graduate text in Mathematics-52. SpringerVerlag, 1977.

[Otw03] A. Otwinowska. Composantes de petite codimension du lieu de Noether-Lefschez: un argument asymptotique en faveur de la conjecture de Hodge. J. Alg. Geom., 12(2):307$320,2003$.

[Otw04] A. Otwinowska. Sur les varietes de Hodge des hypersurfaces. arXiv:math/0401092v1 [math.AG], 2004.

[Ser06] E. Sernesi. Deformaions of Algebraic Schemes. Grundlehren der mathematischen Wissenschaften-334. Springer-Verlag, 2006.

[Voi88] C. Voisin. Une précision concernant le théorème de Noether. Math. Ann., 280(4):605$611,1988$.

[Voi89] C. Voisin. Composantes de petite codimension du lieu de Noether-Lefschetz. Comm. Math. Helve., 64(4):515-526, 1989.

[Voi02] C. Voisin. Hodge Theory and Complex Algebraic Geometry-I. Cambridge studies in advanced mathematics-76. Cambridge University press, 2002.

[Voi03] C. Voisin. Hodge Theory and Complex Algebraic Geometry-II. Cambridge studies in advanced mathematics-77. Cambridge University press, 2003. 